\title{
ELABORACIÓN DE UN PERFIL DE LA ORIENTACIÓN EN EDUCACIÓN SECUNDARIA DESDE LAS DEMANDAS DE LA COMUNIDAD EDUCATIVA
}

\author{
ELABORATION OF A GUIDANCE PROFILE IN SECONDARY EDUCATION \\ BASED ON THE DEMANDS OF THE EDUCATIONAL COMMUNITY
}

\author{
Ignacio González López* \\ Universidad de Córdoba \\ y Francisco Javier García Rueda** \\ IES La Escribana
}

\section{RESUMEN}

Este trabajo busca delimitar, a partir de un estudio empírico realizado en Córdoba capital y provincia, cuáles son las necesidades planteadas al departamento de orientación por parte de la familia, el alumnado, el profesorado, los equipos directivos y la inspección, con el fin de ayudar a los orientadores y orientadoras a seleccionar y promover entre sus múltiples tareas aquellas que son más demandadas. Del mismo modo, puede servir como base para una posible regulación de la función orientadora en los centros de secundaria.

Palabras clave: Orientación educativa, orientación profesional, orientación personal, educación secundaria, comunidad educativa

\section{ABSTRACT}

From an empirical study developed in Cordova and province, the study presented in this article seeks to highlight the needs expressed by families, pupils, teaching staff, headteachers, and inspectors in relation to the work of the Guidance Department, with the aim of helping guidance workers to select,

\footnotetext{
* Ignacio González López es Profesor Contratado Doctor del Área de Métodos de Investigación y Diagnóstico en Educación de la Universidad de Córdoba. Sus líneas de investigación se centran en la evaluación de programas educativos y en la medición educativa. E-mail edlgoloi@uco.es

** Francisco Javier García Rueda es Orientador y Director del IES La Escribana de Villaviciosa de Córdoba, así como Especialista en Orientación e Intervención Educativa por el Colegio de Psicólogos de Andalucía Occidental.
} 
among their multiple tasks, those that are most demanded. At the same time, it could serve as the basis for regulating the functions of guidance in secondary schools.

Key words: Educational guidance, career guidance, personal guidance, secondary education, school community.

\section{Introducción}

En 1992, Juan Fernández Sierra se preguntaba sobre qué se esperaba de los orientadores en los centros de secundaria y cual sería su aportación a los mismos. Hoy en día la pregunta sigue en el aire y esa ha sido la finalidad de este estudio, intentar dar respuesta a las expectativas que se tienen desde cada agente educativo con respecto a la labor que realiza un orientador $\mathrm{u}$ orientadora en un centro de educación secundaria.

A ello le sumamos las múltiples funciones y demandas que le adjudican, entre las que destacan la atención a la diversidad, la evaluación y promoción del alumnado, la educación para la salud, la educación en valores, la atención a situaciones de acoso escolar, la atención a problemas de disciplina, el tratamiento del fracaso escolar, atención en situaciones de desmotivación, incorporación de innovaciones metodológicas y materiales didácticos, etc. (Orden de 17 de julio de 1995), o clasificadas en una serie de competencias como el diagnóstico, orientación educativa, desarrollo de la carrera, counseling, información, consulta, investigación, gestión de programas y servicios, desarrollo comunitario y empleo (Asociación Internacional de Orientadores Educativos y Profesionales, 2003), todo ello sin una orden de la Consejería de Educación de la Junta de Andalucía que actualice las funciones y competencias de los departamentos de orientación desde 1995.

Por otra parte, no podemos olvidar que el profesional de la orientación de un centro de Educación Secundaria, sólo en la etapa obligatoria es responsable de aproximadamente unos 200 alumnos y alumnas, o que el de un Instituto de Educación Secundaria trabaja directamente con 1200 alumnos y alumnas de Educación Secundaria Obligatoria, Bachillerato, Formación Profesional de grado medio y superior y Programas de Garantía Social.

Todo esto lleva a que muchos profesionales realicen sus múltiples funciones sin más criterio que el suyo propio o el que el equipo directivo estime oportuno, sin tener en cuenta muchas veces las necesidades más inmediatas que tienen los distintos agentes educativos (profesorado, alumnado, familias, equipos directivos e inspección).

Son numerosos los estudios que vienen a solicitar un perfil de la orientación en educación secundaria así como la revisión y adaptación de la formación que reciben tanto los alumnos y alumnas de psicopedagogía como los orientadores y orientadoras en activo. Álvarez Rojo et al. (1992) plantean que "en un periodo corto de tiempo se ha pasado en la orientación de carencia absoluta de experiencia a una situación en las que se le pide a sus profesionales una eficacia que, cuando menos, produce desconcierto por su magnitud y experiencia". A ello se le suman las manifestaciones de Estebaranz y Mingorance (2001), quienes consideran que "los orientadores van construyendo socialmente sus roles en cada contexto, en función de lo que consideran que deben hacer ante las necesidades que perciben, y de su concepción de las tareas del orientador, pero también de la percepción y aceptación y demanda que el resto de la comunidad tiene de su función". Yendo más allá, Veláz de Medrano et al. (2001) afirman que "el ejercicio de la profesión en un contexto de refor- 
ma profunda (y continua) del sistema educativo, supone unas dificultades y demandas adicionales dirigidas a los profesionales de la docencia y la orientación".

Por otro lado, Royo (2002) presenta un trabajo sobre el desempeño profesional de los orientadores en los institutos de educación secundaria de Salamanca, donde descubrió que estos profesionales manifestaban una serie de preocupaciones personales-profesionales relacionadas con la multitud de funciones que se les atribuyen, la asignación de un horario elevado de docencia restando tiempo a su actividad orientadora, la impartición de materias fuera de su especialidad, la falta de dotación personal en los Departamentos de Orientación, la invisibilidad de sus tareas y las críticas y los rechazos procedentes de otros agentes educativos.

De todo ello cabe señalar que si queremos que la orientación contribuya a la calidad de la enseñanza deben revisarse las atribuciones que se han ido asignando desde su aparición en los centros para evitar una sobrecarga de obligaciones que dificulten el desarrollo del trabajo de estos profesionales. Del mismo modo, es fundamental delimitar aquellas que son prioritarias o imprescindibles para la comunidad educativa.

Es preciso señalar que la orientación se introduce en los centros de secundaria con la finalidad de favorecer la calidad y mejora de la enseñanza, tal y como señala el título cuarto de la LOGSE (1990). Es por ello que, para alcanzar dicho fin, debe partir de la delimitación de unos objetivos y principios de actuación que vertebren este trabajo en los centros, y en los cuales debemos basarnos a la hora de construir el perfil profesional de la orientación.

En definitiva, la amplitud de funciones y competencias justifica la necesidad de establecer unas prioridades de actuación, atendiendo no solamente al marco normativo sino a las demandas de los usuarios de este servicio.

\section{Método}

La finalidad que persigue este estudio consiste en conocer los elementos de la práctica de la orientación que, desde la valoración de los agentes implicados, son considerados más relevantes para adquirir las competencias adecuadas para el desempeño de nuestras funciones en los institutos de educación secundaria, en función de las demandas reales. De este modo, pretendemos identificar y valorar las demandas orientadoras de los diferentes agentes educativos: alumnado, profesorado, familias, equipos directivos y servicios de inspección para, posteriormente, establecer un modelo funcional de necesidades que sirva para determinar el perfil profesional del orientador u orientadora partiendo de las demandas planteadas por los diferentes agentes educativos presentes en este estudio.

Una vez aclarado el propósito que rige este estudio, se especifican las variables que dan cuenta de los fenómenos a estudiar. Para su selección, hemos considerado aquellos elementos que definen las competencias a asumir por el orientador con cada uno de los agentes educativos considerados en este estudio. Las variables han sido agrupadas en las siguientes dimensiones: Disciplina, Didáctica, Hábitos y técnicas de estudio, Tutoría, Atención a la diversidad, Evaluación/promoción, Familia, Clima afectivo, Normativa, Relaciones personales, Programas y Orientación Educativa y Profesional.

La muestra estuvo formada por 275 sujetos de la ciudad de Córdoba y su provincia, distribuidos entre los siguientes agentes educativos (ver tabla 1): 
TABLA 1: Muestra.

\begin{tabular}{|l|c|c|}
\hline \multicolumn{1}{|c|}{ Muestra } & N & \% \\
\hline Inspectores & 3 & 1.1 \\
\hline Directores & 14 & 5.1 \\
\hline Profesorado & 65 & 23.63 \\
\hline Alumnado & 152 & 55.27 \\
\hline Familias & 41 & 14.9 \\
\hline Total & $\mathbf{2 7 5}$ & $\mathbf{1 0 0}$ \\
\hline
\end{tabular}

Como instrumentos de recogida de información se confeccionaron una serie de encuestas autoadministradas (protocolos estandarizados formados por ítems de valoración escalar) ${ }^{1}$ dirigidas a cada uno de los agentes educativos implicados, validados empíricamente ${ }^{2}$, y donde los datos cuantitativos obtenidos se analizaron mediante diferentes técnicas estadísticas y los datos cualitativos se analizaron mediante técnicas del análisis de contenido.

\section{Resultados}

El análisis de los datos obtenidos se presenta estructurado en dos grandes bloques (estudio descriptivo y estudio factorial), atendiendo en todo momento al grupo muestral o agente educativo del cuál se ha extraído la información, con el objeto de generar un perfil competencial de la figura del profesional de la orientación en educación secundaria obligatoria asociado a cada demandante.

\section{Resultados del estudio descriptivo}

La realización del primero de los procesos, descriptivo e inferencial, ha aportado los primeros resultados. Estos se presentan en función de las diferentes dimensiones planteadas en los instrumentos e intentando aportar diferencias según el grupo muestral implicado en cada momento:

- Disciplina: el alumnado y las familias piden intervención por parte del orientador u orientadora, sobre todo a través de la mediación. El profesorado solicita información sobre estrategias de resolución de conflictos para poder aplicar por ellos mismos. Los equipos directivos demandan a los orientadores participación en estrategias de resolución de conflictos y colaboración con la jefatura de estudios.

1. Escala de cuatro valores (1: valor mínimo y 4: valor máximo) que indican el grado de prioridad / necesidad de cada una de las funciones propuestas

2. Un análisis de consistencia interna aportó un valor alfa de Cronbach para los instrumentos superior a 0.84 , a excepción del destinado a la inspección, donde el valor obtenido fue de 0.453 , valor relativamente bajo debido a la escasez de inspectores que participaron en el estudio. Por su parte, el poder de discriminación de los ítems medido por una prueba de $\mathrm{t}$ de Student entre las medias de los grupos alto y bajo reveló que el $90 \%$ de los ítems de cada instrumento es válido. 
- Hábitos y técnicas de estudio: sorprendentemente, el alumnado considera fundamental esta tarea del orientador u orientadora, aún cuando en la práctica es rechazada. Las familias piden asesoramiento sobre cómo contribuir al desarrollo de dichos hábitos.

- La acción tutorial es una de las tareas de la orientación consideradas importantes para el alumnado. El 80\% del profesorado demanda a este profesional la elaboración y participación en las sesiones de tutoría. La acción tutorial para los padres y madres es fundamental y por eso demandan a los orientadores y orientadoras su participación y colaboración con los tutores.

- La atención a la diversidad es una función imprescindible del departamento de orientación para todos los agentes educativos.

- Clima afectivo: el alumnado demanda un clima de confianza dentro de las aulas y es por ello que solicita la intervención de los orientadores y orientadoras para intentar alcanzarlo. Para el profesorado y los equipos directivos, este profesional debe contar con unas adecuadas habilidades sociales para poder desarrollar su trabajo. El orientador/a es pieza clave para la familia en el desarrollo de un buen clima en el centro.

- Evaluación/promoción: la variabilidad normativa en cuanto a este aspecto se refiere a que todos los agentes educativos consideran necesario que exista una persona que les mantenga informados y asesorados, recayendo sobre la figura de este profesional. Los equipos directivos, aunque manifiestan estar informados sobre la normativa, sí solicitan a los orientadores y orientadoras asesoramiento.

- Normativa: en lo referente a esta dimensión, el alumnado se centra especialmente en los aspectos relacionados con la oferta educativa y profesional. El profesorado considera importante que el orientador/a esté informado de la legislación educativa y, a su vez, le mantenga informado.

- El desarrollo personal como objetivo de la educación, se convierte para el alumnado en una función primordial para la orientación. Las familias son conscientes de la etapa evolutiva en la que se encuentran sus hijos e hijas, es por ello que consideran imprescindible la intervención de la orientación en alcanzar este objetivo dentro del centro.

- Programas de orientación: dentro de los diversos programas que se desarrollan en un centro, los de Educación para la Salud (drogodependencias, afectivo-sexual) son los más demandados, seguidos de los de técnicas de trabajo intelectual. Para el profesorado, los programas que más demanda al departamento de orientación son los de técnicas de estudio y habilidades sociales. Los programas de educación para la salud son los más demandados por las familias.

- Dentro de la orientación profesional, al alumnado le interesa fundamentalmente que los orientadores y orientadoras le ayuden a tomar decisiones acertadas. El profesorado no considera primordial que el orientador/a asesore a los departamentos didácticos sobre la inclusión de programas de la orientación en los respectivos currículos. Informar, asesorar y ayudar en la toma de decisiones son las funciones que las familias demandan a estos profesionales con respecto a la orientación profesional.

- Aspectos didácticos: el profesorado no considera como una de las acciones prioritarias del departamento de orientación. Asesorar sobre los principios didácticos que deben contemplar las distintas programaciones didácticas es la función primordial para los equipos directivos. 
- Participación de las familias: el profesorado es consciente de la importancia que ésta tiene sobre la educación del alumnado, por ello demanda como función importante de los orientadores y orientadoras fomentar la participación y comunicación con las familias. Para las familias, el departamento de orientación es el encargado de poner en práctica todas aquellas acciones que sirvan para potenciar su implicación. Para los equipos directivos está claro que si en un centro alguien tiene que dinamizar y potenciar la participación de los padres y madres, así como organizar su formación, estos son los orientadores u orientadoras.

Con respecto al servicio de inspección, destacar que todas las dimensiones sobre las que se les preguntó consideraron que eran funciones imprescindibles de los departamentos de orientación, lo cual, además de coincidir con la regulación legal, aporta poca información.

Seguidamente, se instó a las diferentes audiencias a platear aquellas otras demandas al departamento de orientación que no estuviesen presentes entre las planteadas en las diferentes partes de las encuestas. Dada la escasez de respuestas aportadas a esta pregunta, exponemos las diferentes manifestaciones tal y como han sido presentadas:

- El alumnado únicamente planteó la demanda de "dar consejos sobre los estudios", aspecto que se puede integrar directamente entre las funciones que componen la dimensión de orientación educativa y profesional.

- El profesorado manifestó otra serie de demandas que giran en torno a los aspectos metodológicos y didácticos innovadores a aprender; en la insistencia en la prevención de drogodependencias, que tal y como se ha mostrado anteriormente, es un de los programas prioritarios a desarrollar desde la perspectiva del propio alumnado; y opinan que en la orientación educativa y profesional debería darse una mayor implicación de todos los que intervienen en la formación del alumnado (padres, equipos educativos, orientador, etc.) a partir del desarrollo de programas de información y ayuda en la toma de decisiones.

- Son las familias las que más demandas plantearon, más allá de las presentadas en la encuesta. Consideraron relevante, al igual que el profesorado y el alumnado, desarrollar programas educativos sobre drogas. Por su parte, centraron sus demandas en aspectos disciplinarios como enseñar al alumnado a resolver los problemas que ocurren dentro y fuera del aula, proponiendo en su caso medidas sancionadoras y ejemplares, hacer responsable al alumnado de la limpieza del centro educativo y dotar de libertad al profesorado sobre medidas disciplinarias.

- El equipo directivo consideró que es primordial dar estabilidad o continuidad al orientador u orientadora a petición del equipo directivo, así como que los centros con 20 unidades, incluida la post-obligatoria, cuenten con dos profesionales.

- Desde la inspección no se plantearon nuevas demandas.

\section{Resultados del estudio factorial}

Aproximarnos de un modo más certero y válido al perfil del orientador partiendo de las necesidades demandadas por los diferentes agentes educativos es una de las metas de la técnica que ahora se propone, el análisis factorial. 
El análisis comienza, una vez establecidos los métodos de extracción (componentes principales) y de rotación (varimax), seleccionando las variables que hacen referencia al concepto genérico que estamos estudiando: el perfil profesional de los profesionales de la orientación desde la perspectiva de los diferentes agentes educativos: alumnado, profesorado, familias, equipo directivo. Dada la limitación de acceso a un número más elevado de inspectores e inspectoras, la aplicación de esta técnica no ha podido llevarse a cabo. Se establecen, de este modo, cinco propuestas tal y como se presenta en la siguiente tabla (ver tabla 2).

Como puede apreciarse, se han obtenido una totalidad de 24 elementos que definen con precisión cuáles son las funciones que ha de cumplir un orientador y que son la base para la definición de su perfil profesional. Con una media de doce funciones obtenidas a partir de las demandas de cada agente educativo, y con una explicación media de la varianza del $79.21 \%$, lo cual verifica la explicación de la mayoría de la totalidad de la variabilidad del criterio, observamos una serie de elementos comunes en estos agentes como son:

- Asesoramiento al alumnado en la toma de decisiones.

- Implicación de las familias en la educación de sus hijos.

- Asesoramiento normativo sobre evaluación/promoción.

- Participación en el diseño y desarrollo de las tutorías.

- Orientación personal.

- Elaboración de normas de convivencia en el centro.

- Elaboración de normas de convivencia en el centro.

- Propuesta de nuevas medidas disciplinarias.

- Diagnóstico de necesidades educativas especiales.

Estas tareas pueden ser las que sirvan para la elaboración de un único perfil de la labor orientadora, a pesar de que en este trabajo se pretenden analizar las demandas de los diferentes agentes educativos $\mathrm{y}$, por lo tanto, extraer diferentes perfiles asociados a cada uno de ellos.

En definitiva, los factores conseguidos que tratan de aportar una explicación del abanico de competencias de los orientadores y las orientadoras, desde cada agente educativo, son los siguientes:

\section{Alumnado}

- Factor 1: Ayuda al alumnado sobre el proceso de toma de decisiones.

- Factor 2: Implicación de las familias en la educación de sus hijos.

- Factor 3: Orientación profesional.

- Factor 4: Asesoramiento normativo sobre evaluación/promoción.

- Factor 5: Conocimiento de la oferta educativa existente.

- Factor 6: Asesoramiento al docente sobre orientación y currículum.

- Factor 7: Participación en el diseño y desarrollo de las tutorías.

- Factor 8: Información sobre hábitos y técnicas de estudio. 
Elaboración de un perfil de la orientación en Educación...

Ignacio González et al.

TABLA 2: Factores obtenidos y porcentaje de varianza explicada para cada agente educativo.

\begin{tabular}{|c|c|c|c|c|}
\hline \multirow{3}{*}{ Factores } & \multicolumn{4}{|c|}{ Agentes educativos } \\
\hline & $\begin{array}{l}\frac{0}{0} \\
\frac{0}{c} \\
\frac{c}{2} \\
\frac{D}{4}\end{array}$ & $\begin{array}{l}\frac{0}{0} \\
\frac{\pi}{2} \\
\frac{0}{0} \\
\frac{\Phi}{0} \\
\frac{0}{0}\end{array}$ & 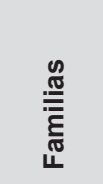 & 을 \\
\hline & \multicolumn{4}{|c|}{ Varianza explicada } \\
\hline Ayuda al alumnado en el proceso de toma de decisiones & 7.016 & 8.676 & 5.856 & \\
\hline Implicación de las familias en la educación de sus hijos & 6.443 & 5.574 & 7.228 & \\
\hline Orientación profesional & 6.234 & & 10.13 & \\
\hline Asesoramiento normativo sobre evaluación/promoción & 5.855 & 3.995 & 6.039 & \\
\hline Conocimiento de la oferta educativa existente & 5.088 & & & \\
\hline Asesoramiento al docente sobre orientación y currículum & 4.926 & & & \\
\hline Participación en el diseño y desarrollo de las tutorías & 4.543 & 4.668 & 7.982 & \\
\hline Información sobre hábitos y técnicas de estudio & 4.443 & & 4.174 & \\
\hline Orientación personal & 4.384 & 5.439 & 6.318 & \\
\hline Desarrollo de programación de educación para la salud & 4.113 & 8.719 & 7.698 & \\
\hline Elaboración de normas de convivencia en el centro & 4.044 & 4.178 & 5.190 & \\
\hline Propuesta de nuevas medidas disciplinarias & 3.939 & 3.302 & & 15.14 \\
\hline Diagnóstico de necesidades educativas especiales & 3.828 & 3.814 & & 8.504 \\
\hline Fomento de un clima afectivo adecuado & & 6.520 & 8.088 & \\
\hline Asesoramiento sobre legislación educativa & & 6.439 & & 7.195 \\
\hline Atención a la diversidad & & 5.992 & & 10.74 \\
\hline Asesoramiento sobre investigación e innovación educativa & & & & \\
\hline Asesoramiento sobre metodologías didácticas & & 5.068 & & 6.751 \\
\hline Mediación en los problemas de convivencia & & & 7.332 & \\
\hline Atención individualizada en la orientación profesional & & & 5.300 & \\
\hline Elaboración de programas de diversificación curricular & & & & 18.88 \\
\hline Asesoramiento didáctico sobre concreción curricular & & & & 11.84 \\
\hline Fomento de las relaciones con el exterior & & & & 10.67 \\
\hline Asesoramiento sobre recursos para la docencia & & & & 9.003 \\
\hline Varianza total explicada & 64.86 & 77.92 & 81.33 & 92.73 \\
\hline
\end{tabular}


- Factor 9: Orientación personal.

- Factor 10: Desarrollo de programación de educación para la salud.

- Factor 11: Elaboración de normas de convivencia en el centro.

- Factor 12: Propuesta de nuevas medidas disciplinarias.

- Factor 13: Diagnóstico de necesidades educativas especiales.

\section{Profesorado:}

- Factor 1: Desarrollo de programas de educación para la salud.

- Factor 2: Ayuda al alumnado sobre el proceso de toma de decisiones.

- Factor 3: Fomento de un clima afectivo adecuado.

- Factor 4: Asesoramiento sobre legislación educativa.

- Factor 5: Atención a la diversidad.

- Factor 6: Implicación de las familias en la educación de sus hijos.

- Factor 7: Asesoramiento sobre investigación e innovación educativa.

- Factor 8: Orientación personal.

- Factor 9: Asesoramiento sobre metodologías didácticas.

- Factor 10: Participación en el diseño y desarrollo de las tutorías.

- Factor 11: Elaboración de normas de convivencia en el centro.

- Factor 12: Asesoramiento normativo sobre evaluación/promoción.

- Factor 13: Diagnóstico de necesidades educativas especiales.

- Factor 14: Propuesta de nuevas medidas disciplinarias.

\section{Familias}

- Factor 1: Orientación profesional.

- Factor 2: Fomento de un clima afectivo adecuado.

- Factor 3: Participación en el diseño y desarrollo de las tutorías.

- Factor 4: Desarrollo de programas de educación para la salud.

- Factor 5: Mediación en los problemas de convivencia.

- Factor 6: Implicación de las familias en la educación de sus hijos.

- Factor 7: Orientación personal.

- Factor 8: Asesoramiento normativo sobre evaluación/promoción.

- Factor 9: Ayuda al alumnado sobre el proceso de toma de decisiones.

- Factor 10: Atención individualizada en la orientación profesional.

- Factor 11: Elaboración de normas de convivencia en el centro.

- Factor 12: Información sobre hábitos y técnicas de estudio. 


\section{Equipos directivos}

- Factor 1: Propuesta de nuevas medidas disciplinarias.

- Factor 2: Elaboración de programas de diversificación curricular.

- Factor 3: Asesoramiento didáctico sobre concreción curricular.

- Factor 4: Atención a la diversidad.

- Factor 5: Fomento de las relaciones con el exterior.

- Factor 6: Asesoramiento sobre recursos para la docencia.

- Factor 7: Diagnóstico de necesidades educativas especiales.

- Factor 8: Asesoramiento sobre legislación educativa.

- Factor 9: Asesoramiento sobre metodologías didácticas.

\section{Conclusiones}

Según los trabajos elaborados por Sobrado (1997), Repetto, Ballesteros y Malik (1999) y Cano (2003), tanto desde la perspectiva epistemológica como desde el análisis de la práctica de trabajo, la orientación contiene como notas dominantes la amplitud y la diversificación de funciones y tareas, resultando complejo identificarla con una realidad única y concreta. La cuestión se agrava desde el momento en que se adopta una óptica transnacional. El dominio de actuación de la orientación se amplía y se diversifica cada vez con mayor fuerza.

Con ello, se exige de la orientación, como de cualquier otra rama profesional, una adaptación a esta nueva realidad. A través de la formación adecuada se puede lograr el ajuste que se pretende.

Cualquier profesional de la orientación educativa que lea este estudio pensará que estos resultados ya los sabía, pero si lee detenidamente los datos obtenidos podrá descubrir "curiosidades" que nos pueden permitir encauzar nuestro trabajo desde ese perfil demandado por alumnado, profesorado, familias, equipos directivos e inspección. Con esto queremos decir que la elaboración del presente estudio nos ha enseñado, además de lo complicado que es en todos los niveles la investigación, que el trabajo de la orientación en secundaria debe prestar más atención a las demandas que nos hacen, algunas en silencio, todos los miembros de la comunidad educativa. Solo así se contribuirá a la calidad de la enseñanza y no exclusivamente a un cúmulo de tareas realizadas "por decreto".

Muchas veces, la falta de información o de una estructura que organice tu trabajo hace que éste sea difícil de desarrollar. De ahí la importancia de tener reguladas las funciones y competencias de la orientación, pero puede ocurrir que esa información sea tan amplia y abierta que dé cabida a multitud de campos de actuación, con lo cual esta labor, aún siendo los regulada la administración, carezcan de significatividad para los "usuarios" de este servicio.

Por ello, es importante conocer, averiguar y analizar cuáles son las demandas que ellos tienen, con el objeto de poder concretar y priorizar nuestras funciones en base a un análisis de necesidades específico. Eso es lo que ha pretendido este trabajo, delimitar aquello que es 
más importante para cada uno de los agentes que intervienen en el proceso educativo, extrayendo tras su análisis, un perfil que nos sirva de guía.

El objetivo de este trabajo era delimitar las acciones más demandas por los distintos agentes que intervienen en el proceso educativo con respecto al departamento de orientación, con el objeto de delimitar un perfil profesional. Sin embargo, los resultados obtenidos nos llevan a la delimitación de cuatro perfiles, uno por cada agente, exceptuando la inspección, que debido a la muestra tan limitada con la que hemos contado y que en sus demandas no existe ninguna priorización, ha sido imposible extraer conclusiones significativas.

Dentro de las demandas que se hace de cada sector, éstas han sido definidas en base al grado de relevancia obtenido del análisis factorial efectuado al efecto y que, presentadas de forma priorizada, son la base para la confección de estos perfiles.

\section{Perfil del orientador u orientadora a partir de las demandas del alumnado}

Las funciones más demandadas por este sector de la población se refieren, en primer lugar, a aquellas que inciden sobre la toma de decisiones y, por consiguiente, al desarrollo de programas que faciliten esa madurez vocacional que les lleve a tomar decisiones acertadas en cuanto a su futuro profesional se refiere. A continuación, el alumnado pide acciones que aumenten la implicación de las familias en su educación, una necesidad tan acuciante en la época que vivimos, que llama la atención que hasta los propios alumnos y alumnas la demanden. Unido a la toma de decisiones se solicita información y asesoramiento relacionado con la oferta educativa y profesional, así como de los aspectos normativos y recursos que la administración dispone. También es destacable que soliciten al orientador u orientadora que sea un asesor del profesorado, tanto en aspectos curriculares como de orientación. Otro factor relevante para ellos es la participación de estos profesionales en el diseño y desarrollo de la acción tutorial, así como los hábitos y técnicas de estudio. A continuación, el alumnado demanda acciones que vayan dirigidas a desarrollar estilos de vida saludable, buena convivencia en el centro, incluyendo la mediación y propuestas de medidas que la favorezcan. Por último, asesoramiento al equipo docente en cuestiones relativas a la atención de las dificultades de aprendizaje.

\section{Perfil del orientador u orientadora a partir de las demandas del profesorado}

La prioridad del profesorado en cuanto a las funciones de la figura responsable de la orientación en un centro comienzan por el desarrollo de programas de educación para la salud, ayuda al alumnado en su toma de decisiones, fomento de un clima adecuado dentro del centro, asesoramiento sobre legislación educativa, coordinar la atención a la diversidad, actuaciones en pro de una mayor participación familiar en la educación, asesoramiento sobre investigación e innovación educativa y asesoramiento sobre metodologías didácticas, lo cual contrasta con la idea de que presentan reticencia a recibirlo. Las tutorías, tanto en su diseño como en su desarrollo, es otra de las funciones que deben asumir estos profesionales, al igual que participar en las normas de convivencia del centro, diagnosticar las dificultades de aprendizaje y asesorar sobre evaluación. 


\section{Perfil del orientador u orientadora a partir de las demandas de las familias}

Las familias esperan del orientador y la orientadora que desarrolle la orientación profesional de sus hijos, favorezca un clima afectivo dentro del centro, coordine el diseño y desarrollo de las tutorías, dinamice programas de educación para la salud, intervenga en las normas de convivencia del centro, fomente su propia implicación en la educación, desarrolle programas que incidan sobre su desarrollo personal, les informe sobre las novedades que regulan la educación en cuanto a legislación se refiere, ayude a sus hijos a tomar decisiones acertadas, que la orientación profesional tenga una actuación lo más individualizada posible $\mathrm{y}$, por último, quieren que les informe sobre cómo favorecer hábitos y técnicas de estudio adecuadas.

\section{Perfil del orientador u orientadora a partir de las demandas de los equipos directivos}

Los equipos directivos de los centros de secundaria tienen un caballo de batalla que es cómo afrontar los problemas de convivencia que se dan en los centros, y es por ello que su principal demanda hacia el orientador $\mathrm{u}$ orientadora se centra en el asesoramiento sobre medidas disciplinarias, seguidas de aspectos relacionados con la atención a al diversidad como son la elaboración de programas de diversificación curricular; también solicitan asesoramiento sobre los principios didácticos que deben establecerse en las programaciones, especialmente en lo referente a los temas transversales y la atención a la diversidad desde el aula. En este profesional ven al responsable de establecer contactos con los recursos del exterior. Al igual que ocurría con el profesorado, también es ésta la persona que puede asesorarles en cuestiones metodológicas, didácticas y legislativas y, por supuesto, es la encargada de evaluar las necesidades educativas especiales del alumnado.

Finalmente, se ha comprobado que las funciones a las que este profesional atiende en función de las demandas que se le hacen son muy numerosas y variadas, pero habría que modificar la estructura que actualmente existe en la organización de la orientación en los centros de secundaria. Nos referimos concretamente a la ratio orientador/alumnos. Si a las numerosas funciones le sumamos que estamos en un centro de mil estudiantes, nuestro trabajo sólo puede ceñirse a un modelo estandarizado de actuación. Igualmente, podrían incluirse en el currículo formativo de psicopedagogía o en la formación continua contenidos relacionados con la mediación y estrategias de resolución de conflictos, dinamización de las familias, programas operativos para el desarrollo de la toma de decisiones, habilidades sociales y atención a la diversidad.

\section{Referencias biliográficas}

Alvárez Rojo, V., García Jiménez, E., Rodríguez Diéguez, A. y Cruz Martínez, J. M. (1992). “El perfeccionamiento de los orientadores en ejercicio en el contexto de la reforma: una cuestión pendiente". Qurrículum, 5, 85-100.

Asociación Internacional de Orientación Educativa y Profesional (2003). Competencias Internacionales para los Profesionales de Orientación y de Educación. Aprobado para la Asamblea General de la AIOEP. Berna, 4 de septiembre de 2003. 
Cano, J. (2003). "Diversidad de roles del orientador psicopedagógico: posibilidades y dificultades en su trabajo profesional". Anuario de pedagogía, 5, 103-119.

Estebaranz, A. y Mingorance, P. (2001). "La construcción de la función orientadora en los centros de secundaria. La visión de sus protagonistas". Revista de la Facultad de Ciencias de la Educación, 3, 79-94.

Fernández Sierra, J. (1992). El trabajo docente y psicopedagógico en educación secundaria. Málaga: Aljibe.

LOGSE (1990). Ley Orgánica 1/1990, de 3 de octubre, de Ordenación General del Sistema Educativo (Boletín Oficial del Estado número 238, de 4 de octubre de 1990).

Orden de 17 de julio de 1995, por la que se establecen las directrices sobre la organización y funciones de la acción tutorial del profesorado y los Departamentos de Orientación (Boletín Oficial de la Junta de Andalucía número 117, de 29 de agosto de 1995).

Repetto, E.; Ballesteros, B. y Malik, B. (1999). "Hacia una formación de los orientadores en Europa. Estudio empírico de las áreas de competencias más relevantes". Revista Española de Orientación y Psicopedagogía, 10 (17), 149-162.

Royo, F. (2002). "Desempeño profesional de los orientadores en los institutos de educación secundaria de Salamanca. Creación de un instrumento de valoración y autovaloración”. Revista Española de Orientación y Psicopedagogía, 13 (1), 31-50.

Sobrado, L. (1997). "Evaluación de las competencias profesionales de los orientadores escolares". Revista de Investigación Educativa, 15 (1), 83-102.

Velaz de Mendrano, M. C., Blanco, A., Guillamón, J. R., Repetto, E., Torrego, J. C. y Negro, A. (2001). "El desarrollo profesional de los orientadores de educación secundaria: Análisis de necesidades y prospectiva". Revista de Investigación Educativa, 19 (1), 199-220.

Fecha de recepción: 05-04-06

Fecha de revisión: 23-05-07

Fecha de aceptación: 07-11-07 